\title{
Oncology patient-reported claims: maximising the chance for success
}

\author{
H Kitchen ${ }^{1}$ D Rofail' ${ }^{1}$, M Caron ${ }^{2}$ and M-P Emery ${ }^{2}$ \\ ${ }^{1}$ Mapi Values, Cheshire, UK \\ ${ }^{2}$ Mapi Research Trust, Lyon, France \\ Correspondence to H Kitchen. Email: helen.kitchen@mapivalues.com
}

\begin{abstract}
Objectives/purpose: To review Patient Reported Outcome (PRO) labelling claims achieved in oncology in Europe and in the United States and consider the benefits, and challenges faced.

Methods: PROLabels database was searched to identify oncology products with PRO labelling approved in Europe since 1995 or in the United States since 1998. The US Food and Drug Administration (FDA) and the European Medicines Agency (EMA) websites and guidance documents were reviewed. PUBMED was searched for articles on PRO claims in oncology.

Results: Among all oncology products approved, 22 were identified with PRO claims; 10 in the United States, 7 in Europe, and 5 in both. The language used in the labelling was limited to benefit (e.g. "....resulted in symptom benefits by significantly prolonging time to deterioration in cough, dyspnoea, and pain, versus placebo") and equivalence (e.g. "no statistical differences were observed between treatment groups for global QoL"). Seven products used a validated HRQoL tool; two used symptom tools; two used both; seven used single-item symptom measures (one was unknown). The following emerged as likely reasons for success: ensuring systematic PRO data collection; clear rationale for pre-specified endpoints; adequately powered trials to detect differences and clinically significant changes; adjusting for multiplicity; developing an a priori statistical analysis plan including primary and subgroup analyses, dealing with missing data, pooling multiple-site data; establishing clinical versus statistical significance; interpreting failure to detect change. End-stage patient drop-out rates and cessation of trials due to exceptional therapeutic benefit pose significant challenges to demonstrating treatment PRO improvement.
\end{abstract}

Conclusions: PRO labelling claims demonstrate treatment impact and the trade-off between efficacy and side effects ultimately facilitating product differentiation. Reliable and valid instruments specific to the desired language, claim, and target population are required. Practical considerations include rationale for study endpoints, transparency in assumptions, and attention to subtle variations in data.

Published: 09/05/2011

Received: 07/03/2011

ecancer 2011, 5:212 DOI: 10.3332/ecancer.2011.212

Copyright: $\odot$ the authors; licensee ecancermedicalscience. This is an Open Access article distributed under the terms of the Creative Commons Attribution License (http://creativecommons.org/licenses/by/2.0), which permits unrestricted use, distribution, and reproduction in any medium, provided the original work is properly cited. 


\section{Background}

Patient Reported Outcome (PRO) questionnaires are useful in assessing any aspect of patients' health status (disease and care) from their perspective [1]. They cover single and multi-dimensional concepts including, but not limited to, symptoms (e.g. nausea, dyspnoea), functioning, health-related quality of life (HRQoL), treatment satisfaction, and adherence [2]. Essentially, PROs provide an important perspective on how patients feel and function that cannot usually be adequately captured by clinical measures [3].

Although there are some evidence to suggest a relationship between tumour response and PRO data [4] traditionally, oncology clinical trial endpoints have focused on clinical measures such as tumour response rate and time to tumour progression (TTP) [5]. Nevertheless, improvement in long-term survival rates in some cancers, such as testicular [6] and recurrent breast cancer [7], have increased the need for long-term treatments for which impact upon patient symptoms and HRQoL is a key concern.

Recently, regulatory agencies, the US Food and Drug Administration (FDA) and European Medicines Agency (EMA), emphasised the importance of PRO data and issued guidance on the incorporation of PRO instruments into clinical trials [1,8]. Including PRO endpoints in phase II and III clinical trials for oncology products can demonstrate treatment impact from the patient's perspective and make substantial contributions to the awareness of the disease burden on patients. PRO data can also provide significant prognostic information beyond standard clinical measure [9-11].

\section{Methods}

The PROLabels database was searched to identify oncology products with PRO labelling which had been approved in Europe since 1995 or in the United States since 1998. The FDA and EMA websites and guidance documents were reviewed. In addition, PUBMED was searched for relevant articles using the following keywords: 'oncology' OR 'cancer' AND 'patient-reported outcomes' AND 'claim', 'label' or 'regulatory'.

Data were extrapolated from the Drug Product Label and the Summary Base Approval (SBA) (as available) including: the brand and generic product name, date of approval, label indication, type of PRO claim (e.g. HRQoL, symptoms, functioning), PRO claim language, PRO instruments used, and PRO endpoint status. The PRO instruments used to obtain labelling claims were reviewed in light of FDA PRO guidance [12].

\section{Results}

\section{PRO label claims granted in oncology products by FDA and EMA}

A review of the PROLabels website (http://www.mapi-prolabels.org) revealed over 125 oncology products that were approved by FDA and/or EMA between 1995 and 2009. Of these, 22 were identified with PRO claims; 10 in the United States, 7 in Europe, and 5 in both (Table 1).

The majority of the label claims approved by both the FDA and EMA were based on signs and symptoms: six products obtained a label claim for pain, six products obtained a label claim for emesis and five products obtained a label claim for nausea. The language used in label claims was limited to showing benefit and equivalence (Table 2)

\section{PRO questionnaires used to support label claims in oncology products}

Looking at the label claims that have been granted after FDA draft guidance in 2006 (Table 3), a diverse range of instruments have been utilised to assess the impact of treatment upon patient symptoms and aspects of functioning, from single-item visual analogue scales to multidimensional questionnaires such as the Functional Assessment of Cancer Therapy-Lung (FACT-L). 
Table 1: PRO label claims granted in oncology products by FDA and EMA, 1995-2010

\begin{tabular}{|c|c|c|c|c|c|c|c|c|c|c|}
\hline \multirow[b]{2}{*}{ Year } & \multicolumn{5}{|l|}{ FDA } & \multicolumn{4}{|l|}{ EMA } & \multirow[b]{2}{*}{$\begin{array}{l}\text { All oncology } \\
\text { approvals }\end{array}$} \\
\hline & HRQoL & $\begin{array}{l}\text { Signs and } \\
\text { symptoms }\end{array}$ & $\begin{array}{l}\text { Multiple } \\
\text { labels }\end{array}$ & $\begin{array}{l}\text { Any } \\
\text { PRO }^{\dagger}\end{array}$ & $\begin{array}{c}\text { All } \\
\text { oncology } \\
\text { approvals }\end{array}$ & HRQoL & $\begin{array}{l}\text { Signs and } \\
\text { symptoms }\end{array}$ & $\begin{array}{l}\text { Multiple } \\
\text { labels }\end{array}$ & $\begin{array}{l}\text { Any } \\
\text { PRO' }\end{array}$ & \\
\hline \multicolumn{11}{|c|}{ Pre-draft guidance } \\
\hline 1995-1996 & & 2 & 1 & & - & & 1 & 1 & & 5 \\
\hline $1997-1999$ & & 1 & & 1 & 10 & 1 & & & & 8 \\
\hline $2000-2002$ & 1 & 1 & & & 11 & & & & & 14 \\
\hline 2003-2005 & & 1 & 1 & 1 & 13 & & 2 & 2 & & 10 \\
\hline \multicolumn{11}{|c|}{ Post-draft guidance } \\
\hline 2006-2008 & 1 & 1 & & & 19 & & & & & 23 \\
\hline \multirow[t]{2}{*}{$2009-2010^{\ddagger}$} & & \multirow[t]{2}{*}{1} & \multirow[t]{2}{*}{1} & & \multirow[t]{2}{*}{7} & 2 & 1 & \multirow[t]{2}{*}{1} & & \multirow[t]{2}{*}{8} \\
\hline & & & & & & 1 & 4 & & & \\
\hline Total & 2 & 7 & 3 & 2 & 60 & 4 & 10 & 4 & & 68 \\
\hline
\end{tabular}

\section{Table 2: Language used in PRO label claims}

\begin{tabular}{|l|l|}
\hline Benefit & Equivalence \\
\hline $\begin{array}{l}\text { "...resulted in symptom benefits by significantly prolonging time to deterioration in cough, } \\
\text { dyspnoea, and pain, versus placebo". }\end{array}$ & $\begin{array}{l}\text { "no statistical differences were observed between } \\
\text { treatment groups for global QoL". }\end{array}$ \\
\hline $\begin{array}{l}\text { "Quality of life outcomes differed according to EGFR mutation status. In EGFR mutation- } \\
\text { positive patients, significantly more IRESSA-treated patients experienced an improvement in } \\
\text { quality of life and lung cancer symptoms vs carboplatin/paclitaxel”. }\end{array}$ & - \\
\hline $\begin{array}{l}\text { "The mean sum of pain intensity differences at } 30 \text { minutes (SPID30) for ONSOLIS-treated } \\
\text { episodes was statistically significantly higher than for placebo-treated episodes". }\end{array}$ & - \\
\hline
\end{tabular}

A review of the PROs used to obtain label claims in oncology products revealed that, in light of FDA guidance, they are generally not 'fit for purpose' i.e. the majority of the instruments were developed without input from the intended treatment population.

\section{Discussion}

\section{Challenges in obtaining PRO label claims in oncology}

There are a number of potential challenges involved in achieving a PRO label claim for oncology products. First, the FDA guidance states "Generally, findings measured by a well-defined and reliable PRO instrument in appropriately designed investigations can be used to 
Table 3: PRO instruments used to support label claims, post 2006

\begin{tabular}{|c|c|c|c|}
\hline Instrument & Claim & Regulatory agency & Division \\
\hline \multicolumn{4}{|l|}{ Signs and symptoms label claims } \\
\hline \multirow[t]{3}{*}{ VAS } & Pain & EMA & \multirow{3}{*}{$\begin{array}{l}\text { CDER - Division of Gastroenterology } \\
\text { Products }\end{array}$} \\
\hline & Nausea & EMA & \\
\hline & Nausea & FDA & \\
\hline LCSS & Symptoms & EMA & \\
\hline SPID30 & Pain & FDA & $\begin{array}{l}\text { CDER - Division of Anesthesia, } \\
\text { Analgesia, and Rheumatology Products }\end{array}$ \\
\hline Symptom Scale Assessment & Symptom Palliation & EMA & \\
\hline \multicolumn{4}{|c|}{ Health related quality of life label claims } \\
\hline EORTC QLQ-C30 & HRQoL & EMA & \\
\hline FLIE & $\begin{array}{l}\text { Impact of chemotherapy-induced nausea and } \\
\text { vomiting on patients' daily lives }\end{array}$ & FDA & $\begin{array}{l}\text { CDER - Division of Gastroenterology } \\
\text { Products }\end{array}$ \\
\hline FACT-L & HRQoL & EMA & \\
\hline
\end{tabular}

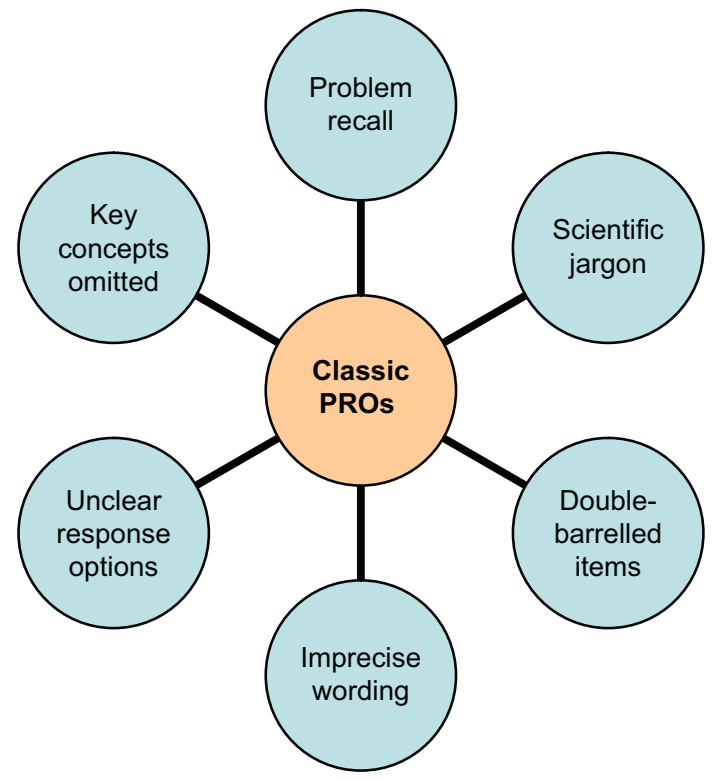

Figure 1: Common problems with classic PRO measurements.

support a claim in medical product labelling if the claim is consistent with the instrument's documented measurement capabilities" [12]. This requires content validity to be established, including qualitative research, to ensure that items measure the intended concept, are relevant, comprehensive and understood by the intended population. PRO instruments should also be reliable, responsive, and able to detect change in the intended treatment population. 


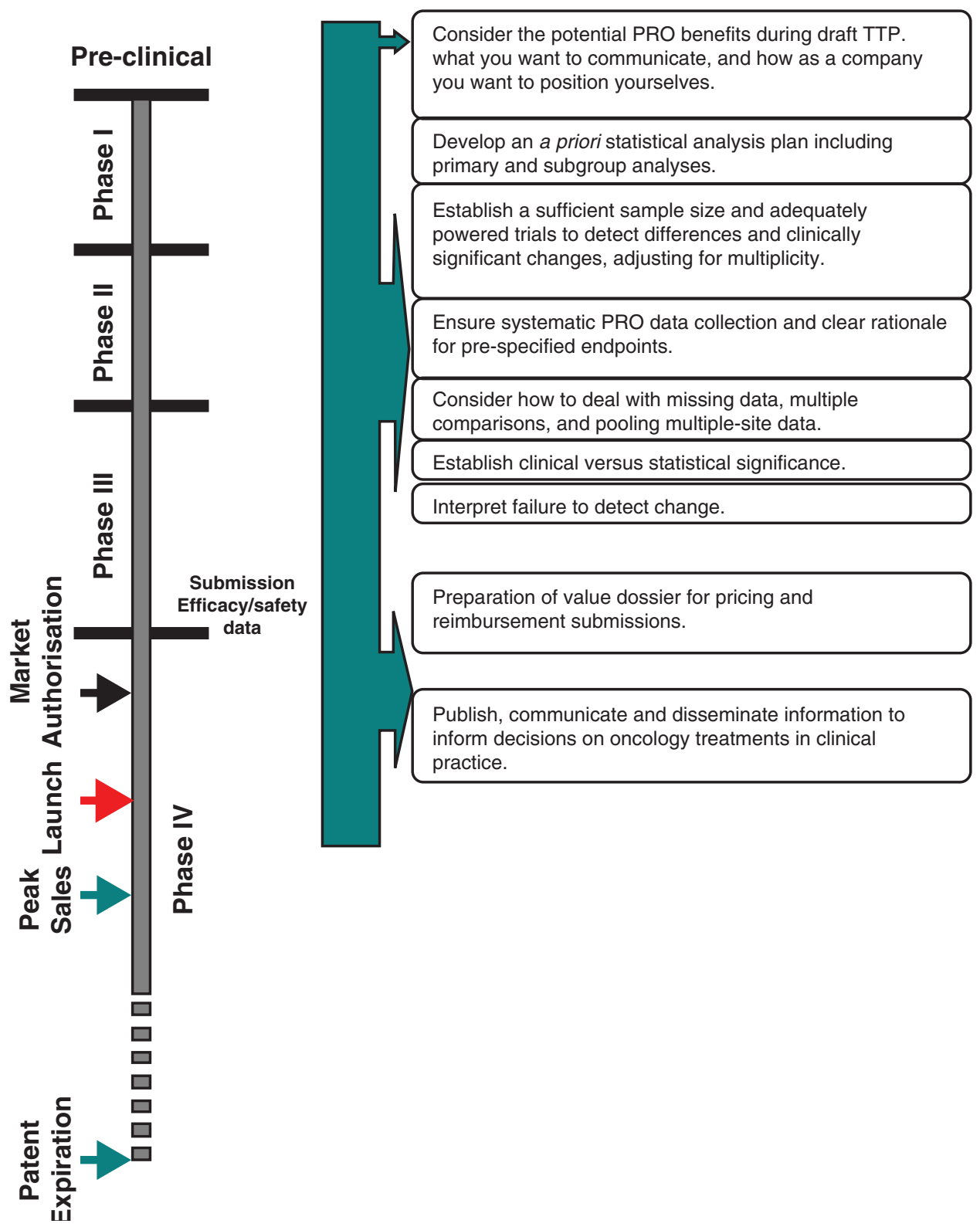

Figure 2: Strategic considerations across an anti-cancer product life-cycle.

Figure 1 shows further common problems with 'classic' PRO instruments including inappropriate recall period and imprecise wording which can be problematic for patients to complete, therefore threatening the consistency and validity of data obtained.

Furthermore, there are specific challenges associated with oncology clinical trials, in particular the complexity of demonstrating symptom or HRQoL improvement in an indication where patients get progressively worse. Demonstrating long-term impacts of treatments requires the sample to be retained for the duration of the trial. This may be especially difficult in samples of late-stage and end-stage patients. In 
addition, oncology trials are often un-blinded which poses a problem for the accuracy of PRO data which is frequently subject to selection bias and reporting bias [13].

\section{How to maximise chances for success in achieving a PRO label claim}

The following may contribute towards obtaining an oncology PRO label claim:

- ensuring systematic PRO data collection and clear rationale for pre-specified endpoints

- establishing sufficient sample size and adequately powered trials to detect differences and clinically significant changes, adjusting for multiplicity

- developing an a priori statistical analysis plan including primary and subgroup analyses

- establishing 'stringent' missing data criteria, adjusting for multiple comparisons, and being sensitive to pooling multiple-site data

- establishing clinical versus statistical significance with clear a priori responder definitions

- interpreting failure to detect change

These considerations should be taken at various points during the product life-cycle. Figure 2 provides an overview of when consideration of these factors during the product lifecycle is recommended. However, early planning to select, develop, and validate a PRO instrument is advisable in order to overcome these challenges and obtain a PRO label claim for an oncology product.

\section{Study limitations and future recommendations}

This review focussed on the use of PROs in clinical trials where a label claim has been granted. It is important to recognise the limitations that this presents. Due to availability of information in the public domain, it was not possible to assess oncology studies where PRO instruments have been used inappropriately or unsuccessfully. Future research on a study-by-study basis would provide further insight into the successful use of PROs in oncology clinical trials.

This review indicates that PRO instruments can be used to demonstrate the impact of new treatments on symptoms, side effects, functioning, and aspects of overall HRQoL. When incorporated into clinical trials PRO instruments can provide an important assessment of treatment benefit to patients. Further research with patients in disease-specific populations would be useful to investigate key concepts important to patients in order to identify appropriate PRO instruments.

\section{Conclusions}

PRO labelling claims demonstrate treatment impact, and the trade-off between efficacy and side effects ultimately facilitating product differentiation. Reliable and valid instruments specific to the desired language, claim, and target population are required. Practical considerations include rationale for study endpoints, transparency in assumptions, and attention to subtle variations in data.

\section{Acknowledgement}

This work was supported by funding from Mapi Values and Mapi Research Trust.

\section{Conflict of interest}

H Kitchen is employed by Mapi Values, a health outcomes agency that consults with various pharmaceutical companies. D Rofail was employed by Mapi Values and now works for a pharmaceutical company. 


\section{Author contributions}

H Kitchen and D Rofail designed the study. M Caron and M-P Emery conducted the literature searches. H Kitchen and D Rofail summarized the findings. All authors drafted and finalized the manuscript.

\section{References}

1. US Department of Health and Human Services Food and Drug Administration Center for Drug Evaluation and Research (CDER), Center for Biologics Evaluation and REsearch (CBER), Center for Devices and Radiological Health (CDRH) (2009) Guidance for Industry Patient-Reported Outcome Measures: Use in Medical Product Development to Support Labeling Claims http://www.fda.gov/downloads/Drugs/GuidanceComplianceRegulatorylnformation/Guidances/UCM193282.pdf

2. Marquis $P$, Arnould B, Acquadro C (2006) Patient-reported outcomes and health-related quality of life in effectiveness studies: pros and cons Drug Dev Res 67 193-201 doi:10.1002/ddr.20077

3. Burke LB, Kennedy DL, Miskala PH, Papadopoulos EJ, Trentacosti AM (2008) The use of patient-reported outcome measures in the evaluation of medical products for regulatory approval Clin Pharmacol Ther 84 281-3 PMID: 18580868

4. Victorson D, Soni M, Cella D (2006) Metaanalysis of the correlation between radiographic tumor response and patient reported outcomes Cancer 106 494-504 PMID: 16353212

5. Gondek K, Sagnier PP, Gilchrist K, Woolley JM (2007) Current status of patient-reported outcomes in industry-sponsored oncology clinical trials and product labels J Clin Oncol 25 5087-93 PMID: 17991926

6. Sonneveld DJ, Hoekstra HJ, van der Graaf WT, Sluiter WJ, Mulder NH, Willemse PH et al (2001) Improved long term survival of patients with metastatic nonseminomatous testicular germ cell carcinoma in relation to prognostic classification systems during the cisplatin era Cancer 91 1304-15 PMID: 11283931

7. Giordano SH, Buzdar AU, Smith TL, Kau S-W, Yang Y, Hortobagyi GN (2003) Is breast cancer survival improving? Cancer 100 44-52 PMID: 14692023

8. Committee for Medicinal Products for Human Use (CHMP) (2005) Reflection paper on the regulatory guidance for the use of health-related quality of life (HRQL) measures in the evaluation of medicine products EMEA/CHMP/EWP/139391/2004 http://www.ema.europa.eu/docs/en_GB/document library/Scientific guideline/2009/09/WC500003637.pdf

9. Gotay CC, Kawamoto CT, Bottomley A, Efficace F (2008) The prognostic significance of patient-reported outcomes in cancer clinical trials J Clin Oncol 26 1355-63 PMID: 18227528

10. Karvonen-Gutierrez CA, Ronis DL, Fowler KE, Terrell JE, Gruber SB, Duffy SA (2008) Quality of life scores predict survival among patients with head and neck cancer J Clin Oncol 26 2754-60 PMID: 18509185

11. Robinson DWJ, Eisenberg DF, Cella D, Zhao N, de Boer C, DeWitte M (2008) The prognostic significance of patient-reported outcomes in pancreatic cancer cachexia J Support Oncol 6 283-90 PMID: 18724539

12. US Department of Health and Human Services Food and Drug Administration Center for Drug Evaluation and Research (CDER), Center for Biologics Evaluation and Research (CBER), Center for Devices and Radiological Health (CDRH) (2006) Guidance for industry: patient-reported outcome measures: use in medical product development to support labeling claims: draft guidance Health Qual Life Outcomes 479 PMID: 17034633

13. Sridhara R, Chen C, Chi GYH, Griebel DJ (2004) Evaluation of health-related quality-of-life measures in oncology drug product applications: issues and concerns J Biopharm Stat 14 23-5 PMID: 15027498 